\title{
Estimulação elétrica transcutânea para tratamento da dor após cirurgia cardíaca: uma revisão sistemática
}

\author{
Transcutaneous electrical nerve stimulation for pain \\ treatment after cardiac surgery: a systematic review
}

\author{
Ana Paula Silva Oliveira' ${ }^{1}$ \\ Natale Sena Cerqueira ${ }^{2}$ \\ André Luiz Cordeiro ${ }^{3}$ (1) \\ Alberto Manoel Sarkis Oliveira ${ }^{4}$ (1)
Íngara Keisle São Paulo Barretto Miranda ${ }^{5}$ (1)
}

1,2,5Faculdade Nobre (Feira de Santana). Bahia, Brasil. anapaulaoliveirafisio@outlook.com, natsena-fisio@hotmail.com, ingarakeisle@hotmail.com ${ }^{3}$ Autor para correspondência. Escola Bahiana de Medicina e Saúde Pública (Salvador); Faculdade Nobre (Feira de Santana). Bahia, Brasil. andrelisboacordeiro@gmail.com

${ }^{4}$ Escola Bahiana de Medicina e Saúde Pública (Salvador); Faculdade Nobre (Feira de Santana). Bahia, Brasil. fisiosarkis@yahoo.com.br

\begin{abstract}
RESUMO | INTRODUÇÃo: A esternotomia na cirurgia cardíaca é marcada por dor pós-operatória. Os medicamentos analgésicos são amplamente utilizados para aliviar esse sintoma, no entanto, apresentam vários efeitos adversos que variam de acordo com a classe terapêutica do medicamento utilizado. Portanto, o uso da Estimulação Elétrica Nervosa Transcutânea (TENS) aparece como uma alternativa não invasiva e de fácil aplicação para reduzir o uso de drogas em pacientes submetidos à cirurgia cardíaca. OBJETIVOS: Revisar sistematicamente os efeitos da TENS no tratamento da dor em pacientes no pós-operatório de cirurgia cardíaca e sua repercussão nos parâmetros respiratórios. METODOLOGIA: Revisão sistemática utilizando a metodologia PICO e palavras-chave (TENS, dor, pacientes no pós-operatório, cirurgia cardíaca). Foram incluídos estudos publicados que abordaram os efeitos da TENS na dor de pacientes após cirurgia cardíaca e, como variável secundária, seu impacto nos parâmetros respiratórios, publicados em português ou inglês, nos anos de 2008 a 2019. Estudos não randomizados excluídos, relatos de casos, observações clínicas e revisões. Além disso, os operadores booleanos "and" e "or" foram utilizados. RESULTADOS: A busca resultou inicialmente em 265 artigos, dos quais 253 foram excluídos, restando 12 artigos. Ao final, foram incluídos 5 artigos. As amostras variaram de 20 a 120 participantes. Dos cinco estudos selecionados, quatro demonstraram redução no nível de dor após cirurgia cardíaca, variando sua aplicação do primeiro dia ao terceiro dia de pós-operatório. Essa redução da dor favoreceu uma diminuição no uso de drogas como a morfina. Além disso, com a redução da dor, houve uma melhora na força muscular ventilatória e nos volumes e capacidades pulmonares. CONCLUSÃo: Conclui-se que a TENS é uma opção não medicamentosa que pode ser utilizada como adjuvante na redução da dor e, consequentemente, na melhoria da força muscular respiratória, volume e capacidade pulmonar, entre outros parâmetros, em pacientes submetidos à cirurgia cardíaca.
\end{abstract}

PALAVRAS-CHAVE: Cirurgia torácica. Estimulação nervosa elétrica transcutânea. Dor.
ABSTRACT | INTRODUCTION: Sternotomy in cardiac surgery is marked by postoperative pain. Analgesic drugs are widely used to relieve this symptom, however, with several adverse effects that vary according to the drug therapeutic class used. Therefore, the use of Transcutaneous Electrical Nerve Stimulation (TENS) appears as a noninvasive and easily applicable alternative for reducing the use of drugs in patients undergoing cardiac surgery. OBJECTIVE: To systematically review the effects of TENS in the treatment of pain in patients in the postoperative cardiac surgery and its repercussion on respiratory parameters. METHODOLOGY: Systematic review using the PICO methodology and keywords (TENS, pain, patients in the postoperative,cardiac surgery). Published studies that addressed the effects of TENS on the pain of patients after cardiac surgery were included and, as a secondary variable, its impact on respiratory parameters, published in Portuguese or English, in the years 2008 to 2019. Excluded nonrandomized studies, case reports, clinical observations and reviews. In addition, the Boolean operators "and" and "or" were used. RESULTS: The search initially resulted in 265 articles, of which 253 were excluded, 12 articles remained. At the end, 5 articles were included. The search initially resulted in 2165 articles. Of these, 2160 were excluded, of which 5 were selected. The samples ranged from 20 to 120 participants. Of the five studies selected, four demonstrated a reduction in the level of pain after cardiac surgery, varying its application from the first day to the third postoperative day. This reduction in pain favored a decrease in the use of drugs such as morphine. In addition, with the reduction of pain, there was an improvement in ventilatory muscle strength and lung volumes and capacities. CONCLUSION: It is concluded that TENS is a non-drug option that can be used as an adjunct in pain reduction and, consequently, improving respiratory muscle strength, lung volume and capacity, among other parameters, in patients undergoing cardiac surgery.

KEYWORDS: Thoracic surgery. Transcutaneous electrical nerve stimulation. Pain. 


\section{Introdução}

Pacientes submetidos a cirurgia cardíaca experimentam vários procedimentos que geram dor. A presença de drenos, tubos e, principalmente, esternotomia são as principais causas de dor no pós-operatório'. Esse sintoma é uma condição fisiológica que demonstra competência do sistema nervoso central, pois alerta para proteção e afeta as respostas físicas e comportamentais ${ }^{2}$. A dor nociceptiva ocorre nesses indivíduos devido à agressão cirúrgica nos músculos e ossos 3 . Devido à dor, o paciente tende a adotar um padrão mais superficial, o que causa uma diminuição nos volumes e capacidades pulmonares.

O termo nocicepção está relacionado ao reconhecimento de sinais dolorosos pelo sistema nervoso, que formula informações relacionadas ou não à lesão². Ou seja, a dor afeta diretamente um dos mecanismos de defesa do corpo humano por meio desses nociceptores ${ }^{2}$. Devido à dor, o paciente não apresenta tosse efetiva, que é um mecanismo de defesa das vias aéreas inferiores, aumentando o risco de infecções pulmonares e permanência hospitalar ${ }^{4}$.

Essas inúmeras cirurgias cardiovasculares são responsáveis por complicações pulmonares pós-operatórias, e a dor pós-operatória é um dos principais fatores associados 5 . A dor está relacionada a uma diminuição efetiva dos exercícios físicos e um declínio significativo na eficácia da tosse devido ao aumento da pressão intratorácica6 ${ }^{6}$.

O uso de drogas em pacientes no pós-operatório de cirurgia cardíaca é rotineiro, e os analgésicos opióides são os mais utilizados, no entanto, apresentam diversos efeitos adversos, como hipotensão, retenção urinária e constipação, além de depressão respiratória, náusea, vômito e outros. pode comprometer a recuperação pós-operatória7. As evidências mostram que o uso combinado com anti-inflamatórios não esteroidais pode reduzir o consumo de morfina; no entanto, esses agentes não estão livres de efeitos indesejáveis, incluindo a inibição da agregação plaquetária, que pode causar hemorragias. Há também relatos de uso de anestésicos locais que bloqueiam a resposta ao estresse associada aos procedimentos cirúrgicos, no entanto, sua cardiotoxicidade é uma grande preocupação, pois compromete a vida do paciente 8,9 .

Nesse cenário, a estimulação elétrica nervosa transcutânea (TENS) é considerada um tratamento alternativo, pois atua de forma não invasiva e é de fácil aplicação, atuando por estimulação sensorial, gerando efeitos terapêuticos a partir da combinação de frequência e comprimento de onda9,10. Assim, é necessário realizar um levantamento de evidências científicas sobre o uso da TENS, visando à redução de complicações dolorosas após a cirurgia cardíaca e seu impacto nos parâmetros ventilatórios.

O efeito do TENS é baseado na teoria do controle das portas que modula a dor por ativar as vias inibitórias descendentes ${ }^{11}$. Ela também promove a liberação de endorfinas, serotonina e hormônios analgésicos, diminuindo assim aos níveis de citocinas ${ }^{12}$. A TENS é um método extremamente seguro e viavel, sendo possível a aplicação diária com mínimos efeitos adversos ${ }^{13}$.

O presente estudo teve como objetivo revisar sistematicamente os efeitos da TENS no tratamento da dor em pacientes no pós-operatório de cirurgia cardíaca e sua repercussão nos parâmetros respiratórios.

\section{Materiais e métodos}

Trata-se de uma revisão sistemática e a questão norteadora deste estudo foi: "Quais são os efeitos da TENS como forma de tratamento da dor e nos parâmetros ventilatórios em pacientes submetidos à cirurgia cardíaca?". A pesquisa foi estruturada com base na estratégia $\mathrm{PICO}^{14}$ (Quadro 1). 
Quadro 1. Estratégia de pesquisa do PICO

\begin{tabular}{|c|c|c|}
\hline Acrônimo & Descrição & Definição \\
\hline P & Paciente & $\begin{array}{r}\text { Pacientes submetidos à cirurgia cardíaca (cirurgia de } \\
\text { revascularização do miocárdio e troca valvar) }\end{array}$ \\
\hline I & Intervenção & Aplicação de estimulação elétrica transcutânea \\
\hline C & Controle & Pacientes que não receberam a intervenção (TENS) \\
\hline O & Desfechos & Dor e parâmetros ventilatórios \\
\hline
\end{tabular}

As seguintes bases de dados foram sistematicamente pesquisadas: Pubmed, SciELO (Scientific Electronic Library Online), LILACS (Literatura Latino-Americana e do Caribe em Ciências da Saúde) e Science Direct. As palavras-chave foram utilizadas: Cirurgia Torácica, Cirurgia Cardíaca, Estimulação Elétrica Nervosa Transcutânea, Dor, parâmetros ventilatórios, capacidade pulmonar, volume corrente, volume corrente, força muscular; sinônimos e palavras relacionadas adicionados pelos operadores booleanos "AND" e "OR", de acordo com os Descritores em Ciências da Saúde (DeCS). A pesquisa foi realizada de setembro a novembro de 2019.

\section{Critérios de elegibilidade}

Foram selecionados estudos publicados em ensaios clínicos randomizados que abordaram os efeitos da TENS no tratamento da dor e seu impacto nos parâmetros ventilatórios, disponíveis em inglês, português ou espanhol, publicados entre 2008 e 2019, com o objetivo de atualizar o tópico. Estudos não randomizados, relatos de casos, observações clínicas e revisões foram excluídos.

\section{Extração dos dados}

Os artigos coletados através das buscas nas bases de dados foram selecionados por meio do rastreamento dos títulos (primeira etapa), resumos (segunda etapa) e leitura completa (terceira etapa). Em seguida, foi realizada uma leitura exploratória dos estudos selecionados e, posteriormente, leitura seletiva e analítica. Os dados extraídos dos artigos foram sistematizados: autores, título, revista, ano, resumo e conclusões, a fim de possibilitar a obtenção de informações relevantes para a pesquisa.
O processo de seleção, extração de dados dos artigos e identificação de aspectos metodológicos foi realizado por dois revisores independentes. Quando houve alguma discordância entre eles, os revisores leram o artigo inteiro novamente para reavaliação. Se a discordância persistir, um terceiro revisor independente avaliou e tomou a decisão final. A pesquisa seguiu os itens do protocolo PRISMA ${ }^{15}$ para revisões sistemáticas.

\section{Avaliação da qualidade metodológica}

A qualidade metodológica dos estudos foi avaliada de acordo com os critérios da escala PEDro, que pontua 11 itens, a saber: 1 - Critérios de elegibilidade, 2 Alocação aleatória, 3 - Alocação oculta, 4 - Comparação da linha de base, 5 - Indivíduos cegos, 6 - Terapeutas cegos, 7 - Avaliadores cegos, 8 - Acompanhamento adequado, 9 - Intenção de tratar a análise, 10 Comparações entre grupos, 11 - Estimativas pontuais e variabilidade. Os itens são pontuados como presentes ( 1 ) ou ausentes (0), gerando uma soma máxima de 10 pontos, com o primeiro item sem contar.

\section{Resultados}

Foram encontrados doze artigos após a leitura do resumo e títulos, dos quais apenas cinco foram selecionados pelos critérios de inclusão. Aqueles que não usaram a fisioterapia como foco principal do tratamento ou artigos com desenho de revisão de literatura (3), estudos não randomizados (3) ou relatos de caso (1) também foram excluídos do estudo. O fluxograma presente na figura 1 mostra todos os critérios e bancos de dados usados para selecionar os artigos. 
Figura 1. Fluxograma para obtenção de ensaios clínicos randomizados com base na TENS para controle da dor e parâmetros ventilatórios após cirurgia cardíaca

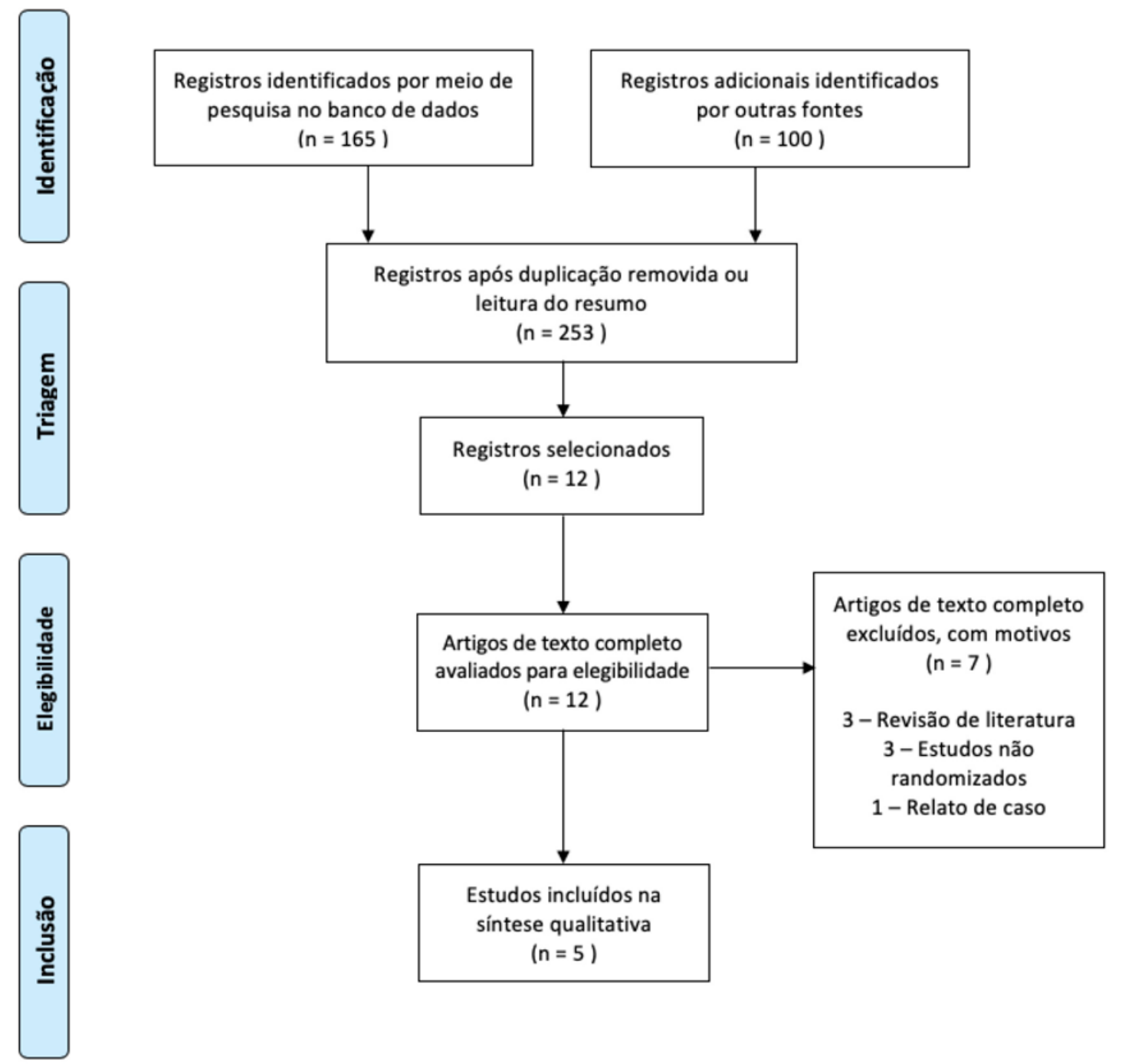

A qualidade metodológica avaliada pela escala PEDro é mostrada na Quadro 2. Os cinco estudos incluídos nesta revisão sistemática discutem o uso da TENS após cirurgia cardíaca, um resumo dos métodos utilizados e os resultados clínicos são apresentados na Tabela 1. 
Quadro 2. Avaliação metodológica da qualidade dos estudos incluídos nesta revisão, utilizando a escala de banco de dados Pedro

\begin{tabular}{|c|c|c|c|c|c|c|}
\hline & & $\begin{array}{l}\text { LIMA,et al } \\
{ }^{16} ., 2011\end{array}$ & $\begin{array}{l}\text { GREGORINI et } \\
\mathrm{al}^{17} ., 2010\end{array}$ & $\begin{array}{l}\text { LUCHESA et } \\
\mathrm{al}^{18}, 2009\end{array}$ & $\begin{array}{l}\text { JUNIOR et } \\
\mathrm{al}^{19}, 2008\end{array}$ & $\begin{array}{l}\text { OZTURK et } \\
\mathrm{al}^{20}, 2016\end{array}$ \\
\hline 01 & $\begin{array}{l}\text { Os critérios de elegibilidade foram } \\
\text { especificados. }\end{array}$ & & & & & \\
\hline 02 & $\begin{array}{l}\text { Sujeitos foram aleatoriamente distribuídos } \\
\text { por grupos. }\end{array}$ & $\checkmark$ & $\checkmark$ & $\checkmark$ & $\checkmark$ & $\checkmark$ \\
\hline 03 & A alocação dos sujeitos foi secreta. & & & & & \\
\hline 04 & $\begin{array}{l}\text { Inicialmente, os grupos eram semelhante no } \\
\text { que diz respeito aos indicadores de } \\
\text { prognósticos mais importantes. }\end{array}$ & & $\checkmark$ & & $\checkmark$ & \\
\hline 05 & $\begin{array}{l}\text { Todos os sujeitos participaram de forma } \\
\text { cega dos estudos. }\end{array}$ & & & & & \\
\hline 06 & $\begin{array}{l}\text { Todos os terapeutas que administram a } \\
\text { terapia fizeram-no de forma cega. }\end{array}$ & $\checkmark$ & & $\checkmark$ & & $\checkmark$ \\
\hline 07 & $\begin{array}{l}\text { Todos os avaliadores que mediram pelo } \\
\text { menos um resultado-chave, fizeram-no de } \\
\text { forma cega. }\end{array}$ & & & & & \\
\hline 08 & $\begin{array}{l}\text { Mensurações de pelo menos um resultado- } \\
\text { chave foram obtidas em mais de } 85 \% \text { dos } \\
\text { sujeitos inicialmente distribuídos pelos } \\
\text { grupos. }\end{array}$ & $\checkmark$ & $\sqrt{ }$ & $\checkmark$ & $\checkmark$ & $\checkmark$ \\
\hline 09 & $\begin{array}{l}\text { Todos os sujeitos a partir dos quais se } \\
\text { apresentaram mensurações de resultados } \\
\text { receberam o tratamento ou a condição de } \\
\text { controle conforme a alocação, ou quando } \\
\text { não foi esse o caso, fez-se a análise dos } \\
\text { dados para pelo menos um dos resultados- } \\
\text { chave por "intenção de tratamento". }\end{array}$ & & & & & \\
\hline 10 & $\begin{array}{l}\text { Os resultados das } \\
\text { comparações estatitisticas inter- } \\
\text { grupos foram descritos pelo menos um } \\
\text { resultado -chave. }\end{array}$ & $\sqrt{ }$ & $\sqrt{ }$ & $\sqrt{ }$ & $\checkmark$ & $\checkmark$ \\
\hline 11 & $\begin{array}{l}\text { O estudo apresenta tanto medidas de } \\
\text { precisão como medidas de variabilidade } \\
\text { para pelo menos um resultado-chave. }\end{array}$ & $\sqrt{ }$ & $\checkmark$ & $\checkmark$ & $\checkmark$ & $\checkmark$ \\
\hline \multicolumn{2}{|c|}{ Pontuação } & $5 / 10$ & $5 / 10$ & $5 / 10$ & $5 / 10$ & $5 / 10$ \\
\hline
\end{tabular}

Luchesa et al. ${ }^{18}$, em pesquisa realizada em 2009, demonstraram a eficácia da TENS na analgesia, embora não tenham sido encontradas diferenças estatisticamente significantes em relação à função respiratória. Por outro lado, em um estudo de Ozturk et al. ${ }^{20}$, uma redução significativa no consumo de morfina foi relatada em um protocolo de analgesia controlada pelo paciente. No entanto, ao comparar os grupos, não foram encontradas diferenças significativas na analgesia entre o grupo controle e o grupo TENS. Os demais autores ${ }^{16,17,19}$ encontraram resultados positivos com o uso da eletroestimulação, promovendo melhora da força muscular, volumes e capacidades pulmonares, como mostra a tabela 1. 


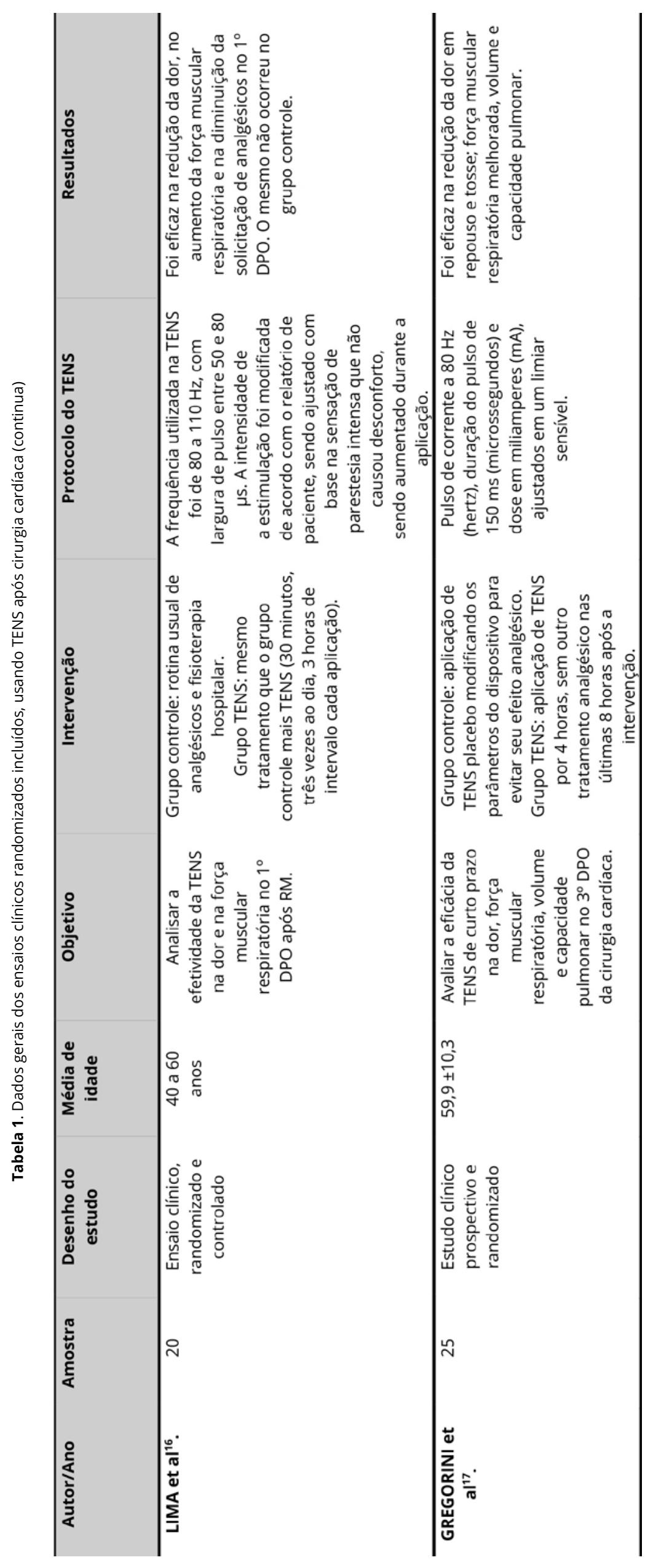




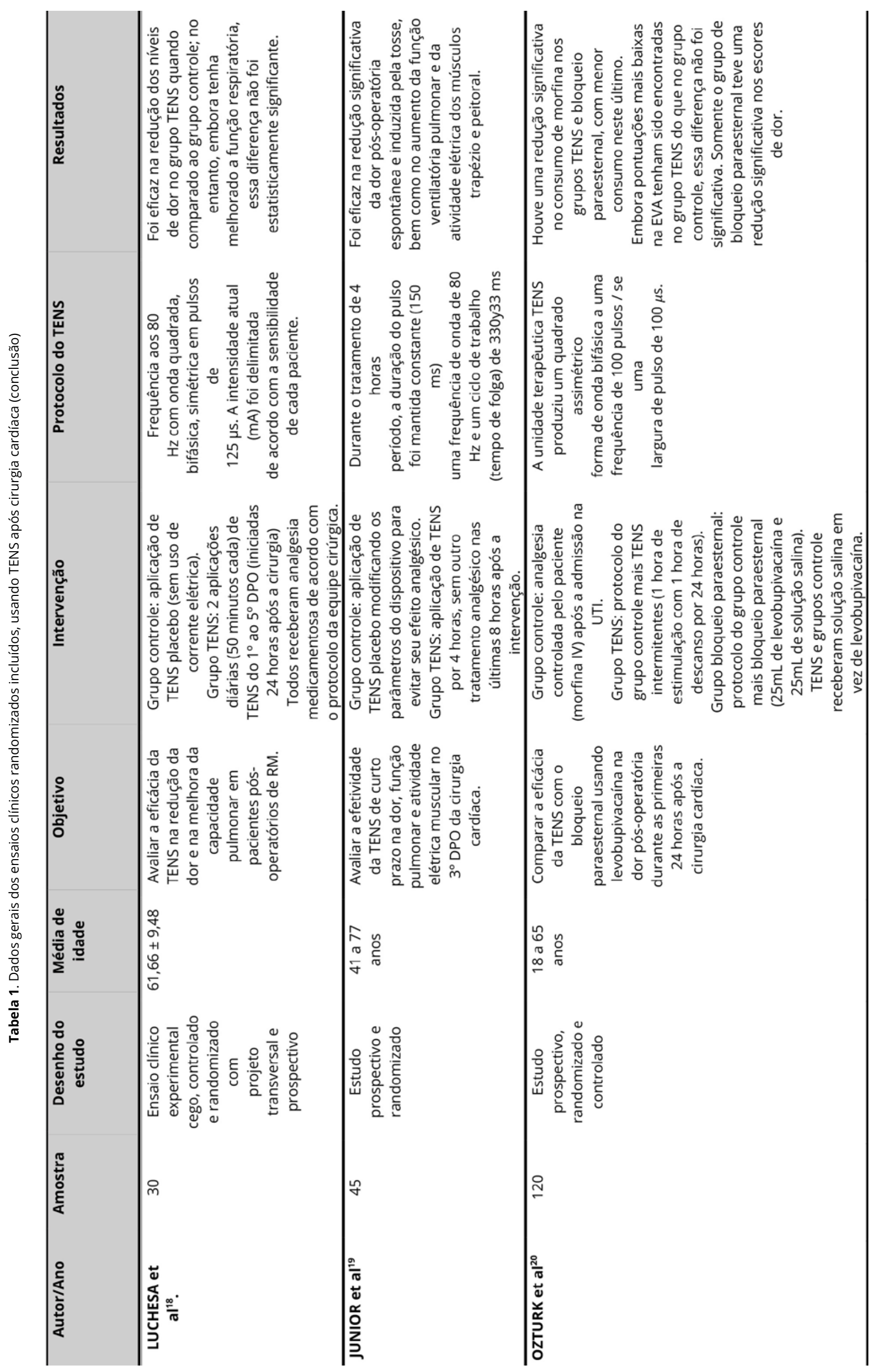




\section{Discussão}

Com base nos resultados obtidos nesta revisão sistemática, pode-se observar que a aplicação da TENS, no pós-operatório de cirurgia cardíaca, é eficaz na redução da dor e, consequentemente, melhora os parâmetros ventilatórios, como volume corrente e capacidade vital.

A TENS é uma intervenção não farmacológica, eficaz na analgesia de condições clínicas agudas e crônicas, como dor nociceptiva pós-operatória, osteoartrite, neuropatia diabética e outras ${ }^{19}$. Seu mecanismo de ação está relacionado a mecanismos centrais e periféricos, incluindo a indução de aumento de beta-endorfinas, encefalina-metionina, bem como a ativação de diferentes receptores opióides, a modulação de mecanismos inibitórios endógenos no sistema nervoso central envolvendo receptores GABA e muscarínico. Alguns efeitos também são mediados por receptores adrenérgicos periféricos ${ }^{19}$.

Em 2015, outro grupo de pesquisa realizou estudos em pacientes submetidos à toracotomia póstero-lateral, demonstrando redução no consumo de analgésicos e diminuição da dor, corroborando os resultados encontrados pelos autores mencionados nesta revisão ${ }^{20}$. Com a diminuição da dor, o paciente tende a não ter medo excessivo de respirar profundamente ou tossir, e esses fatores podem favorecer a melhora da função pulmonar.

Segundo Lima et al. ${ }^{16}$, após a cirurgia, os pacientes apresentam uma involução na força muscular respiratória, favorecendo o aparecimento de complicações pulmonares. Assim, eles propuseram um protocolo cuja intervenção foi o uso de eletroanalgesia em conjunto com o protocolo padrão utilizado para o grupo controle, demonstrando a eficácia do dispositivo na redução da dor e melhora da força muscular.

Especialmente no artigo em que Junior et al. ${ }^{19}$ discutem o uso do dispositivo, o tratamento foi realizado sem divisão de grupo, removendo toda analgesia farmacológica por 8 horas e analisando o efeito da eletroanalgesia sem influência do tratamento farmacológico no resultado desses pacientes. Houve uma diminuição significativa da dor, influenciando diretamente a dor relacionada à tosse, refletindo também melhores parâmetros respiratórios, como volume corrente e capacidade vital.
Como discutido anteriormente, com a diminuição da dor, o paciente começa a apresentar maior expansão torácica, gerando aumento da pressão transpulmonar e aumento da capacidade pulmonar. Além disso, a eletroestimulação pode ser responsável por gerar contração muscular, aumentar a força, tornando o paciente capaz de realizar inspirações mais vigorosas. Essa estimulação pode ocorrer quando alta intensidade é usada. Como discutido anteriormente, com a diminuição da dor, o paciente começa a apresentar maior expansão torácica, negando efetivamente a pressão pleural, gerando aumento da pressão transpulmonar e aumento da capacidade pulmonar. Além disso. Essa estimulação pode ocorrer quando alta intensidade é usada. O uso da TENS tem sido encorajado nestes públicos, uma vez que a utilização de opióides está relacionada ao desenvolvimento de efeitos adversos como sonolência extrema, náuseas, vômitos, supressão da tosse, e maior dificuldade para expectoração ${ }^{21}$.

Por outro lado, os achados de Ozturk et al. ${ }^{20}$ não mostraram analgesia significativa no grupo que recebeu TENS. Neste estudo, houve uma divisão em três grupos distintos, onde parte recebeu bloqueio paraesternal com levobupivacaína, outro grupo recebeu TENS e ambos os grupos e o controle receberam analgesia controlada pelo paciente com morfina. Observou-se que as pessoas submetidas ao bloqueio paraesternal apresentaram melhor eficácia na redução da dor em relação ao grupo que utilizou a TENS no tratamento da dor pós-operatória, o que não apresentou diferença estatística e clínica significantes na analgesia quando comparado ao grupo controle. Os parâmetros de frequência e intensidade de pulso da eletroestimulação devem ser ajustados para alcançar a eficácia analgésica. Entretanto, ao analisar o protocolo utilizado por Ozturk et al. ${ }^{20}$, verificou-se que ele utilizou um protocolo diferente dos demais autores, com parâmetros diferentes, o que pode justificar a ineficácia da eletroanalgesia nessa intervenção.

Diante dos achados relatados nesta revisão sistemática com melhora local da dor na qual a estimulação é aplicada, pode-se inferir que o bem-estar geral do paciente pode ser constatado, refletindo também nos parâmetros de tosse e mobilidade e reduzindo o consumo de drogas, protegendo os sujeitos indivíduos de quaisquer efeitos adversos de tal uso. 
As limitações encontradas nos ensaios clínicos utilizados referem-se à heterogeneidade dos protocolos utilizados, como intensidade e tempo de aplicação. Apesar disso, a aplicação da TENS em pacientes no pós-operatório de cirurgia cardíaca deve ser incentivada, com o objetivo de reduzir a dor e maior adesão ao tratamento proposto. Estudos com a aplicação deste instrumento por um período mais longo e com protocolos mais fechados devem ser incentivados.

\section{Conclusão}

Segundo estudos, a eletroanalgesia através da TENS reduziu a dor em indivíduos submetidos a cirurgia cardíaca. Além disso, foi possível notar que há meIhorias nos parâmetros respiratórios, como aumento de volume e capacidade pulmonar.

\section{Contribuições dos autores}

Cordeiro ALL participou da concepção e delineamento do estudo, análise e interpretação dos dados, redação e revisão e aprovação da minuta final. Oliveira AP, Cerqueira N, Oliveira A, Miranda Participei da concepção e desenho do estudo e da redação do manuscrito.

\section{Conflitos de interesses}

Nenhum interesse financeiro, jurídico ou político concorrente com terceiros (governo, comércio, fundação privada, etc.) foi divulgado para qualquer aspecto do trabalho enviado (incluindo, entre outros, subsídios, conselho de monitoramento de dados, desenho de estudo, preparação de manuscrito, análise estatística, etc.).

\section{Referências}

1. Moss E, Miller CS, Jensen H, Basmadjian A, Bouchard D, Carrier $M$ et al. A randomized trial of early versus delayed mediastinal drain removal after cardiac surgery using silastic and conventional tubes. Interact Cardiovasc Thorac Surg. 2013;17(1):110-5. doi: 10.1093/icvts/ivt123

2. Gosling AP, Oliveira CDC, Périssé F. Neurofisiologia da dor. PROFISIO Esportiva e Traumato-Ortopédica. 2012;1:57-112.

3. Reardon DP, Anger KE, Szumita PM. Pathophysiology, assessment, and management of pain in critically ill adults. Am J Health Syst Pharm 2015;72(18):1531-43. doi: 10.2146/ajhp140541
4. Ögüt S, Sucu Dağ G. Pain Characteristics and Pain Interference Among Patients Undergoing Open Cardiac Surgery. J Perianesth Nurs. 2019;34(4):757-766. doi: 10.1016/j.jopan.2018.10.009

5. Nachiyunde B, Lam L. The efficacy of different modes of analgesia in postoperative pain management and early mobilization in postoperative cardiac surgical patients: A systematic review. Ann Card Anaesth. 2018;21(4):363-370. doi: 10.4103/aca.ACA_186_17

6. Menezes TC, Bassi D, Cavalcanti RC, Barros JESL, Granja KSB, Calles ACDN et al. Comparisons and correlations of pain intensity and respiratory and peripheral muscle strength in the pre- and postoperative periods of cardiac surgery. Rev Bras Ter Intensiva. 2018;30(4):479-486. doi: 10.5935/0103-507x.20180069

7. Huang APS, Sakata RK. Dor após esternotomia --- revisão. Rev Bras Anestesiol. 2016;66(4):395-401. doi: 10.1016/j. bjan.2014.09.003

8. Gardengui G, Kushida CL, Santos JL. Analgesia por Estimulação Elétrica Transcutânea no Pós-operatório de Cirurgia Cardíaca. Revista Brasileira de Saúde Funcional. 2015;2(2):15-23.

9. Shen X, Wang F, Xu S, Qian Y, Liu Y, Yuan H et al. A cardiolipina é o alvo da cardiotoxicidade dos anestésicos locais? Rev Brasileira de Anestesiologia. 2010;60(4):44-454. doi: 10.1590/S0034$\underline{70942010000400013}$

10. Belmonte LM, Brito RM, Machado CL. Efeitos da aplicação da eletroestimulação nervosa transcutânea sobre a dor e volumes pulmonares em indivíduos submetidos á cirurgia cardíaca. $\mathrm{R}$ Fisioter Reab. 2017;1(1):34-41.

11. Dafny N. Pain modulation and mechanisms. In: Neuroscience online. Houston, TX: UTHealth: 2014.

12. Fiorelli A, Morgillo F, Milione R, Pace MC, Passavanti MB, Laperuta $\mathrm{P}$ et al. Control of post-thoracotomy pain by transcutaneous electrical nerve stimulation effect on serum cytokine levels, visual analogue scale, pulmonary function and medication. Eur J of Cardiothorac Surg. 2012;41(4):861-8. doi: 10.1093/ejcts/ezr108

13. Jalalmanesh $M$, Heidari S, Kazemi M, Rahimi-Bashar F, Rostami HR. Effect of transcutaneous electrical nerve stimulation on pain intensity in reduced consciousness patients: A randomized clinical trial. Nursing Practice Today. 2017;4(1):35-43.

14. Santos CMC, Pimenta CAM, Nobre MRC. The PICO strategy for the research question construction and evidence search. Rev Lat-Am Enfermagem. 2007;15(3):508-11. doi: 10.1590/S010411692007000300023

15. Moher D, Liberati A, Tetzlaff J, Altman DG, The PRISMA Group. Preferred Reporting Items for Systematic Reviews and MetaAnalyses: The PRISMA Statement. PLoS Med.2009;6(7):e1000097. doi:10.1371/journal.pmed.1000097 
16. Lima PMB, Farias RTFB, Carvalho ACA, Silva PNC, Filho NAF, Brito RF. Estimulação elétrica nervosa transcutânea após cirurgia de revascularização miocárdica. Rev Bras Cir Cardiovasc 2011;26(4):591-6. doi: 10.5935/1678-9741.20110049

17. Gregorini C, Cipriano Junior G, Aquino LM, Branco JNR, Bernardelli GF. Estimulação Elétrica Nervosa Transcutânea de Curta Duração no Pós-Operatório de Cirurgia Cardíaca. Arq Bras Cardiol 2010;94(3):345-351. doi: 10.1590/S0066$\underline{782 \times 2010000300011}$

18. Luchesa CA, Greca FH, Guarita-Souza LC, Santos JLV, Aquim EE. Papel da eletroanalgesia na função respiratória de pacientes submetidos à operação de revascularização do miocárdio. Rev Bras Cir Cardiovasc. 2009;24(3):391-396. doi: 10.1590/S0102$\underline{76382009000400020}$

19. Cipriano Junior G, Carvalho ACC, Cipriano GFB, Peres PAT. Estimulação elétrica nervosa transcutânea a curto prazo após cardiopatia cirurgia: efeito na dor, função pulmonar e elétrica atividade muscular. Rev Interactive Cardio Vascular and Thoracic Surgery.2008;7(4):539-43. doi: 10.1510/icvts.2007.168542

20. Ozturk NK, Baki ED, Kavakli AS, Sahin AS, Ayoglu RU, Karaveli A et al. Comparação de nervo elétrico transcutâneo Estimulação e Bloqueio Parasternal para Dor no Pós-Operatório Gerenciamento após Cirurgia Cardíaca. Rev Hindawi Publishing Corporation Pain Research and Management. 2016;1(1). doi: 10.1155/2016/4261949

21. Aminisaman J, Mohammadi S, Karimpour H, Hemmatpour B, Sharifi H, Kawyannejad R. Transcutaneous Electrical Nerve Stimulation at the Acupuncture Points to Relieve Pain of Patients Under Mechanical Ventilation: A Randomized Controlled Study. Journal of acupuncture and meridian studies.2018;11(5):290-295. doi: 10.1016/j.jams.2018.06.008 